\title{
A fluorescence-based bioassay for antibacterials and its application in screening natural product extracts
}

\author{
Katharina Michels ${ }^{1}$, Ramona Heinke ${ }^{1}$, Pia Schöne ${ }^{1}$, Oscar P Kuipers ${ }^{2}$, Norbert Arnold ${ }^{1}$ and \\ Ludger A Wessjohann ${ }^{1}$
}

The reliable assessment of the biological activity of a minor component embedded in a complex matrix of several hundred compounds is a difficult but common task in the search for natural product-based antibiotics, for example, by bioassay-guided fractionation. To quantify the antibiotic properties, it is necessary to assess the cell viability. Direct measurements use CFU counts, OD measurements or detection via fluorescent or reducible dyes. However, natural extracts often already possess intrinsic dye, fluorescent, reducing or protein denaturing properties, or they contain insoluble compounds or general protein-binding (tanning) polyphenols as disturbing features, while at the same time very little of the selective antibiotic sought after is present. A promising alternative is provided by intrinsically produced bright fluorescent proteins. In this paper, a rapid, robust and concentration-dependent assay for screening antibiotics with genetically modified mutants of Bacillus subtilis 168 (PabrB-iyfp) is presented. The Gram-positive bacteria exhibit a native fluorescence during their exponential growth phase due to the expression of improved yellow fluorescent protein. To demonstrate the applicability in the field of natural product research, several compounds and extracts were screened for antibacterial activity, with an emphasis on those from the fungal genus Hygrophorus (waxy caps).

The Journal of Antibiotics (2015) 68, 734-740; doi:10.1038/ja.2015.71; published online 8 July 2015

\section{INTRODUCTION}

Biologically active products have been successfully isolated from natural sources, such as plants and fungi, which provide a wide variety of secondary metabolites. Owing to their structural diversity and versatile biological activity, these natural products are valuable lead compounds in pharmaceutical industry. One key step in drug discovery is the assessment of biological activity. A well-established method to evaluate the biological activity is the disk or agar diffusion assay. ${ }^{1-3}$ The procedure relies on the principle of diffusion through a solid growth medium ${ }^{4}$ and is a convenient way to estimate the biological activity of substances and extracts. However, it is not very suitable for quantification of bioactivities, most of all because the diffusion coefficients of the structurally diverse components in such mixtures of hundreds of compounds are not equal. Or if compounds do not diffuse, for example, for lipohilic compounds, even qualitative detection is thwarted. The line dilution assay represents another possibility to assess biological activity. The growth medium is supplemented with a defined concentration of the test substance and growth inhibition is determined subsequently by examination of the cell viability through different direct and indirect detection methods. Two examples of direct methods are cell counting and measuring OD. ${ }^{5,6}$ The former is quite time consuming, whereas the latter suffers from the fact that not only living cells contribute to the OD. Moreover, colored or insoluble/precipitating substances or extracts can disturb the detection, because of strong interfering absorption effects. In contrast to that, the indirect methods employing redox-sensitive dyes such as tetrazolium salts or fluorescent dyes, such as resazurin or fluorescein, allow the selective detection of only viable cells. $^{7-9}$ These assays enable not only the qualitative but also the quantitative determination of bioactivity, but in parallel are associated with some considerable drawbacks influencing their reliability. In case of the colorimetric assay with tetrazolium salts, the strong influence of $\mathrm{pH}$ and possible abiotic reactions with compounds within the assay or extract medium can perturb the method. Furthermore, because this technique also relies on absorption measurements, colored substances and extracts can distort the results. In case of the use of fluorescent dyes, autofluorescence of compounds and extracts is a minor problem, if a specific excitation and emission wavelength can be chosen, but an inconsistent uptake can be a matter of concern. To overcome these difficulties, making use of intrinsically produced fluorescent proteins is a very elegant alternative. ${ }^{10,11}$ It provides the advantage of direct fluorescence measurements for assessment of the cell viability without supplementing any dyes. This enables a very sensitive possibility to examine bioactivity, especially if such a fluorescence is coupled to cell growth and does not persist, for example, in dead cells.

Here, we present a rapid and robust, fluorescence-based bioassay for the determination of antibacterial activity and its application in screening fungal extracts from the genus Hygrophorus.

${ }^{1}$ Department of Bioorganic Chemistry, Leibniz Institute of Plant Biochemistry, Halle (Saale), Germany and ${ }^{2}$ Department of Genetics, Groningen Biomolecular Sciences and Biotechnology Institute, University of Groningen, Groningen, The Netherlands

Correspondence: Professor LA Wessjohann, Department of Bioorganic Chemistry, Leibniz Institute of Plant Biochemistry, Weinberg 3 , 06120 Halle (Saale), Germany.

E-mail: wessjohann@ipb-halle.de

Received 1 July 2014; revised 13 April 2015; accepted 10 May 2015; published online 8 July 2015 


\section{MATERIALS AND METHODS}

Test organism and its cultivation

A genetically modified mutant of the Gram-positive bacterium B. subtilis 168 (PabrB-iyfp) was used as test organism. B. subtilis 168 is the most widely used model strain of $B$. subtilis. It is naturally competent and it is relatively easy to make genetic knockouts and integrations in 168. In this strain, the iyfp gene is under control of the $a b r B$ promoter and its construct and transformation is described in detail elsewhere. ${ }^{12}$ The PabrB-iYFP construct is chromosomally integrated in the amyE locus. ${ }^{12}$ Because it is single copy and chromosomally integrated it is much more stable than when we would have placed it on a multi-copy plasmid. The improved variant relates to the higher production due to improved translation by adding of a heptameric $N$-terminal tail as described by Veening et al. ${ }^{12}$ Sequencing and functional analysis of the genome of B. subtilis strain 168 has been reported, ${ }^{13}$ also effects of gene expression and phosphorelay perturbations on architecture, sporulation and spore resistance. $^{14,15}$

For cultivation and maintenance of the bacterial strain, a complex growth medium ( $1 \%$ tryptone, $0.5 \%$ yeast extract, $1 \% \mathrm{NaCl}$ ) supplemented with chloramphenicol $\left(5 \mu \mathrm{g} \mathrm{ml}^{-1}\right)$ for mutant selection was used. The bacterial strain was maintained on solid tryptone-yeast medium grown over night at $30^{\circ} \mathrm{C}$ and stored at $4{ }^{\circ} \mathrm{C}$.

As inoculum for the antibacterial assay, a preculture was prepared by inoculation of $50 \mathrm{ml}$ tryptone-yeast extract medium in a 100-ml Erlenmeyer flask with one loop CFU taken from a culture grown on solid medium. The preculture was incubated for $24 \mathrm{~h}$ at $30^{\circ} \mathrm{C}$ without shaking. After this time, the cell number was determined using a Neubauer counting chamber and diluted to the appropriate cell number with fresh tryptone-yeast extract medium.

\section{Determination of growth curves}

The growth curves of $B$. subtilis ( $\mathrm{PabrB}$-iyfp) were determined over a period of $40 \mathrm{~h}$ by measurement of $\mathrm{OD}$ at $\lambda_{\mathrm{OD}}=570 \mathrm{~nm}$ and fluorescence intensity at $\lambda_{\mathrm{ex}}=510 \mathrm{~nm}, \lambda_{\mathrm{em}}=535 \mathrm{~nm}$ in microtiter plates. Therefore, the bacterial cultures $(270 \mu \mathrm{l}$ growth medium $+30 \mu \mathrm{l}$ inoculum) were inoculated with a preculture of $1.6 \times 10^{5}, 1.6 \times 10^{6}$ or $1.6 \times 10^{7}$ cells per $\mathrm{ml}$ and incubated for $40 \mathrm{~h}$ at $30^{\circ} \mathrm{C}$. For determination of the growth kinetics, the plates were read out once per hour.

The solvent tolerance was tested by applying 2.5 or 5 or $10 \%$ vol of dimethyl sulfoxide (DMSO) and methanol $(240 \mu$ l growth medium $+30 \mu$ of 25 or 50 or $100 \%$ DMSO, or 25 or 50 or $100 \%$ methanol $+30 \mu \mathrm{l}$ inoculum). Inoculum level in the solvent tolerance experiments was $1.6 \times 10^{5}$ cells per ml.

\section{Dynamic range}

For determination of the dynamic range, a microtiter plate was inoculated with a preculture of $1.6 \times 10^{5}$ cells per $\mathrm{ml}(240 \mu \mathrm{l}$ medium $+30 \mu \mathrm{l} 100 \%$ methanol $+30 \mu \mathrm{l}$ inoculum) for $15 \mathrm{~h}$ at $30^{\circ} \mathrm{C}$. After this time, the cell number was determined using a Neubauer counting chamber. The inoculated bacterial culture was then diluted using fresh tryptone-yeast extract medium supplemented with $10 \%$ vol methanol in 1:2 dilution steps until a dilution of 1:512 was reached. Afterwards, the fluorescence intensity of each dilution step $(n=6)$ was measured.

\section{Assay procedure}

The antibacterial bioassay was carried out in flat-bottom 96-well microtiter plates (BD Falcon/BRANDplates). For measurements of OD, transparent microtiter plates were used, and for better fluorescence detection, black microtiter plates were employed. For inoculation, a $24 \mathrm{~h}$ preculture was prepared and diluted to $1.6 \times 10^{5}$ cells per $\mathrm{ml}$. On each microtiter plate, three different controls $(\mathrm{K})$ were generated. K1 $(n=1)$ : autofluorescence of sample $270 \mu \mathrm{l}$ medium $+30 \mu \mathrm{l}$ sample; K2 $(n=6)$ : bacterial growth control $270 \mu \mathrm{l}$ medium $+30 \mu$ linoculum; K3 ( $n=6$ ): reference (media) control $240 \mu$ l medium $+30 \mu \mathrm{l}$ methanol $+30 \mu \mathrm{l}$ inoculum. K3 was used for all measurements of antibiotics and (crude) fungal extracts as reference control. Furthermore, the reference compound erythromycin was used as positive control K4: $240 \mu \mathrm{l}$ medium $+30 \mu$ l erythromycin diluted in 100\% methanol (final concentrations in wells $=\beta=10^{-4}, 10^{-2}$ or $\left.10^{0} \mu \mathrm{M}\right)+30 \mu \mathrm{l}$ inoculum. Samples $(n=3): 240 \mu \mathrm{l}$ medium $+30 \mu \mathrm{l}$ sample $+30 \mu \mathrm{l}$ inoculum. The samples were applied in three different concentrations $\left(\beta=1 ; 10 ; 100 \mu \mathrm{g} \mathrm{ml}^{-1}\right.$ in methanol) in triplicate. Tetracycline, paromomycin, kanamycin, amoxicillin, spectinomycin and ampicillin were tested analogously to erythromycin in the concentration range from 0.001 to $10 \mu \mathrm{m}$ using fluorescence assay. After inoculation, the fluorescence measurement at time point $t_{0}$ was carried out using a microplate reader GENios Pro (Tecan GeniosPro Reader, Grödig, Austria) at excitation wavelength of $\lambda_{\mathrm{ex}}=510 \mathrm{~nm}$ and emission wavelength of $\lambda_{\mathrm{em}}=535 \mathrm{~nm}$. MeOH has no intrinsic fluorescence effect in this wavelength range. The plates were incubated for $15 \mathrm{~h}$ at $30^{\circ} \mathrm{C}$ without shaking. After this time, the fluorescence intensity $(X)$ was determined again $\left(\lambda_{\mathrm{ex}}=510 \mathrm{~nm}, \lambda_{\mathrm{em}}=535 \mathrm{~nm}\right.$, bandwidth $=10 \mathrm{~nm}$, gain 45 , 60 , 10 flashes, integration time $40 \mu \mathrm{s}, 50 \%$ mirror, $3 \times 3$ quadratic measurements per well, temperature $27^{\circ} \mathrm{C}$, shaking $10 \mathrm{~s}$ low intensity, settle time $1 \mathrm{~s}$ ). The degree of bacterial growth inhibition was then calculated as growth inhibition relative to control K3 (100\% growth) according to Equation (1).

$$
\text { Inhibition }=\left(1-\frac{\overline{\mathrm{X}}_{\text {sample }}\left[t_{15}-t_{0}\right]}{\overline{\mathrm{X}}_{\mathrm{K} 3}\left[t_{15}-t_{0}\right]}\right) \cdot 100 \%
$$

The $Z^{\prime}$-factor ${ }^{16}$ of the assay was calculated according to Equation (2) from the growth inhibition kinetics of erythromycin (Ery; $\beta=1 \mu \mathrm{M}$ ) as positive control with fluorescence intensities and its corresponding (s.d.) at time point $t_{15}$ referred to the media control $\mathrm{K} 3$ as negative control.

$$
\mathrm{Z}^{\prime}=1-\frac{3 \cdot \mathrm{sd}\left(\overline{\mathrm{X}}_{\mathrm{Ery}}\left[\mathrm{t}_{15}\right]\right)+3 \cdot \mathrm{sd}\left(\overline{\mathrm{X}}_{\mathrm{K} 3}\left[\mathrm{t}_{15}\right]\right)}{\left|\overline{\mathrm{X}}_{\mathrm{Ery}}\left[\mathrm{t}_{15}\right]-\overline{\mathrm{X}}_{\mathrm{K} 3}\left[\mathrm{t}_{15}\right]\right|}
$$

\section{Fungal material and preparation of fungal extracts}

An overview of specimens used in this study is given in the supporting information in Supplementary Table 1. Fresh fruiting bodies of different Hygrophorus species were collected and afterwards stored at $-20^{\circ} \mathrm{C}$. Voucher specimens are deposited at the Leibniz Institute of Plant Biochemistry Halle, Germany (IPB).

General procedure for the preparation of fungal extracts is as follows: aliquots $(10 \mathrm{~g})$ of frozen fruiting bodies of Hygrophorus spp. were extracted under ultrasonication for $10 \mathrm{~min}$ at $40^{\circ} \mathrm{C}$ using first $3 \times 10 \mathrm{ml} n$-hexane and afterwards $3 \times 10 \mathrm{ml}$ methanol. If not indicated otherwise, $n$-hexane and methanol extracts refer to extracts of frozen fruiting bodies.

For pulverized lyophilized fruiting bodies, aliquots of $1 \mathrm{~g}$ were extracted with an automated extraction machine (Dionex ASE 200 Accelerated Solvent Extraction) in three cycles (preheating $2 \mathrm{~min}$, heating $5 \mathrm{~min}$, static extraction $10 \mathrm{~min}, 60 \%$ vol. flush, venting $30 \mathrm{~s}$, pressure $70 \mathrm{bar}$, temperature $40^{\circ} \mathrm{C}$, solvent 1: $80 \%$ methanol, solvent 2: $100 \%$ methanol, solvent 3: $100 \% n$-hexane). For the kinetic study of growth inhibition, methanol extract of lyophilized H. pustulatus (coll. 46/03) was used.

The extracts were concentrated to dryness in vacuo. For bioassaying, the extract is redissolved in methanol, additionally it can be passed through Chromabond C18 (500 mg) cartridges with $3 \mathrm{ml}$ methanol (Macherey-Nagel).

\section{RESULTS AND DISCUSSION}

\section{Assay development}

The Gram-positive bacterium strain B. subtilis (PabrB-iyfp) exhibits a native fluorescence during the exponential growth phase due to the expression of an improved variant of the yellow fluorescent protein (YFP). ${ }^{12,14}$ This ability can be used to assess the cell growth directly by the detection of fluorescence intensity (instead of turbidity/OD, which also detects dead cells or insoluble extract components). Veening and Kuipers found no effect on growth or physiology of B. subtilis 168 (type strain widely used in the $B$. subtilis research community) when PabrB-YFP was used. ${ }^{15}$ As B. subtilis usually is not under restriction rules for pathogens, it can even be handled by laboratories not equipped or licensed for the use of pathogens, which applies, for example, for most phytochemical labs. The $a b r B$ promoter $(a b r B$ is a transition state regulator) is regulated itself by the master regulator of sporulation: Spo0A. Thus, only under conditions of sporulationinducing media (harsh low-nutrient conditions) and after the 
transition phase (that is, in stationary phase) this promoter could be influenced. As the assay is employed with exponentially growing cells in rich medium, the abrB promoter will not be affected by natural extracts and will not interfere with fluorescence levels. ${ }^{17}$ Therefore, it allows the sensitive quantification of growth inhibition as a bioactive effect of compounds and extracts. Furthermore, interferences of colored substances present in the extracted matrix are reduced to a minimum because of the specific combination of fluorescence
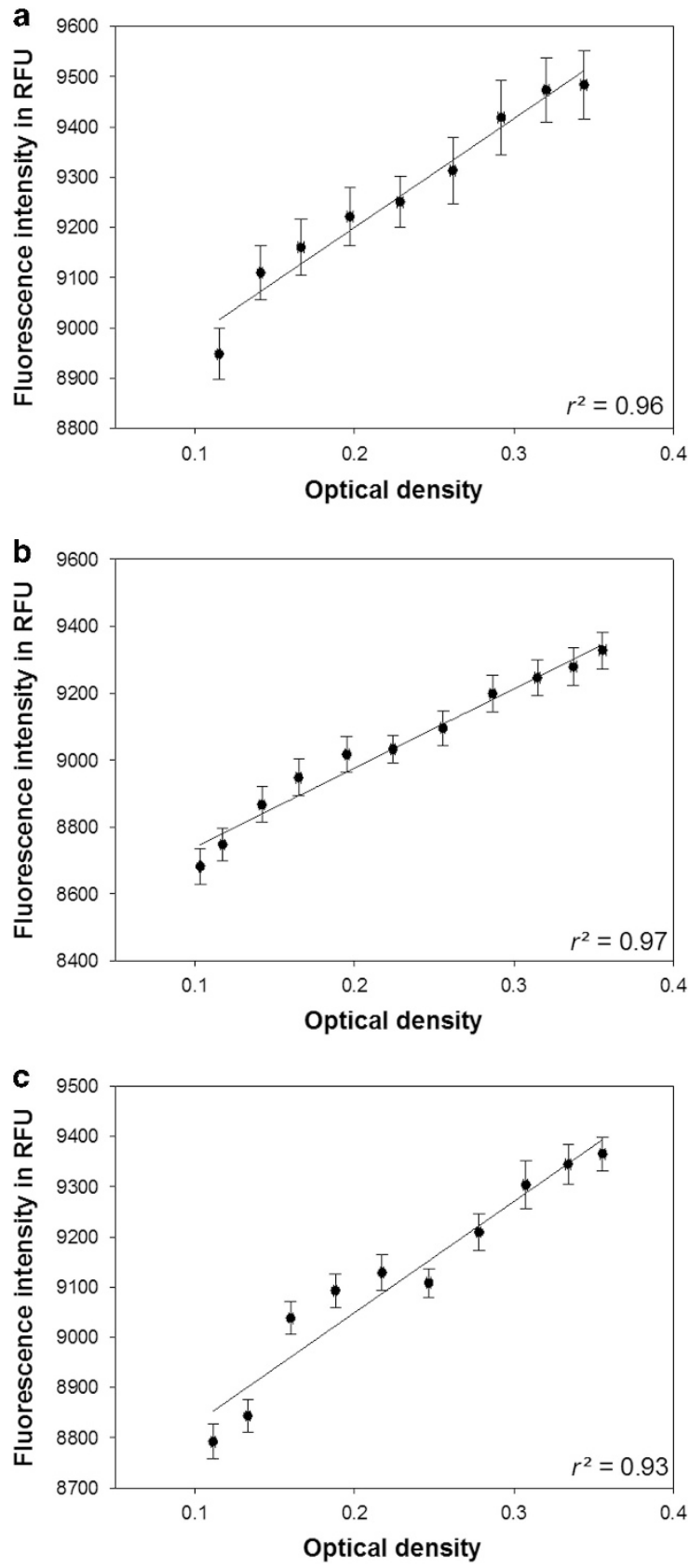

Figure 1 Determination of the linear correlation between $O D$ and fluorescence intensity of growth of $B$. subtilis (PabrB-iyfp) followed by detection of $O D$ and fluorescence intensity in relative fluorescence units (RFU) over time for different cell numbers of the inoculum with corresponding standard errors. (a) $1.6 \times 10^{5}$ cells per ml; (b) $1.6 \times 10^{6}$ cells per $\mathrm{ml}$ and (c) $1.6 \times 10^{7}$ cells per $\mathrm{ml}$. Values at the beginning and after the maximum of fluorescence intensity was reached were excluded (full growth curves see Supplementary Figure 1 in Supplementary Information). excitation and emission wavelengths, thus enabling a reliable and accurate determination of bioactivity. There is neither particular advantage nor disadvantage of using YFP or green fluorescent protein (GFP; admittedly GFP is the most widely used fluorophore). However, significant background fluorescence when using GFP in B. subtilis could be seen and this background is lower when using YFP (this relates to the differences in wavelengths of excitation and emission). Background fluorescence caused by the added antimicrobial medium is also expected to be slightly reduced with YFP. Also in previous assay developments, we found that GFP does not always cease to fluorescence if the expressing organism dies. ${ }^{18}$ This was not yet observed for YFP. Thus, for these two reasons (background and stability) we decided for YFP.

\section{Correlation between OD and fluorescence intensity}

To investigate if there is a direct linear correlation between the bacterial growth and fluorescence intensity, growth curves were determined by measuring turbidity (OD) and fluorescence over time. Therefore, a 24-h preculture was diluted to different cell numbers and applied to the growth assay (Figure 1).

Figure 1a shows the direct linear correlation $\left(r^{2}=0.96\right)$ between the bacterial growth and the fluorescence intensity in a range from $7 \mathrm{~h}$ up to $15 \mathrm{~h}$ observation time in case of an inoculum cell number of $1.6 \times 10^{5}$ (Fluorescence intensity $=(217 \pm 17) \times \mathrm{OD}+877 \pm 4 ; N=9$; $\mathrm{F}=175 ; P<0.0001)$. The linearity holds analogously for an inoculum cell number of $1.6 \times 10^{6}$ cells per $\mathrm{ml}$ in a range of 3 to $13 \mathrm{~h}$ observation time (Figure $1 \mathrm{~b} ; r^{2}=0.97$; Fluorescence intensity $=(237 \pm 14) \times \mathrm{OD}$ $+850 \pm 4 ; N=11 ; \mathrm{F}=311 ; \mathrm{P}<0.0001)$ and for $1.6 \times 10^{7}$ cells per $\mathrm{ml}$ as initial cell number in an observation time ranging from 1 to $10 \mathrm{~h}$ (Figure $1 c ; r^{2}=0.93$; Fluorescence intensity $=(221 \pm 21) \times \mathrm{OD}+861 \pm 6$; $N=10 ; \mathrm{F}=112 ; P<0.0001)$. Moreover, the analyses regarding the influence of the starting cell number demonstrate that the lag-phase observed by measuring OD is also reflected in a delayed increase of fluorescence intensity at the beginning of the experiments. As a consequence, the maximum of the fluorescence intensity is shifted to a later time of growth with decreasing starting cell number (see Supplementary Figure 1 in Supplementary Information). In the late phase, however, OD reaches a maximum or slow growth, whereas fluorescence decreases, indicating that fewer bacteria are in the growth phase. This difference to OD is correct, as YFP is only expressed and persistently active in growth phase and not in resting or dead bacteria. As a conclusion of these experiments, a cell number of $1.6 \times 10^{5}$ cells per $\mathrm{ml}$ was chosen as the standard inoculating concentration and fluorescence measurements were carried out at the beginning and after $15 \mathrm{~h}$ growth time. Furthermore, measurements are performed at the end of the exponential growth phase, which is accompanied with the highest fluorescence intensity and thus signal-to-noise ratio.

Owing to the fact that the majority of natural compounds exhibit only poor water solubility, the addition of an organic solvent to the medium of the assay is essential. Therefore, the solvent tolerance of the fluorescence-based assay with $B$. subtilis was examined by applying different percentages of methanol and DMSO. The tolerance toward both was very different. This phenomenon of strain-dependent solvent tolerance is already known from the literature. ${ }^{19}$ Already $2.5 \%$ vol of DMSO led to a growth inhibition, whereas up to $10 \%$ vol $\mathrm{MeOH}$ can be supplemented without affecting homogeneous growth (Figure 2, up). This compatibility enables the biological testing of many natural products and extracts. 

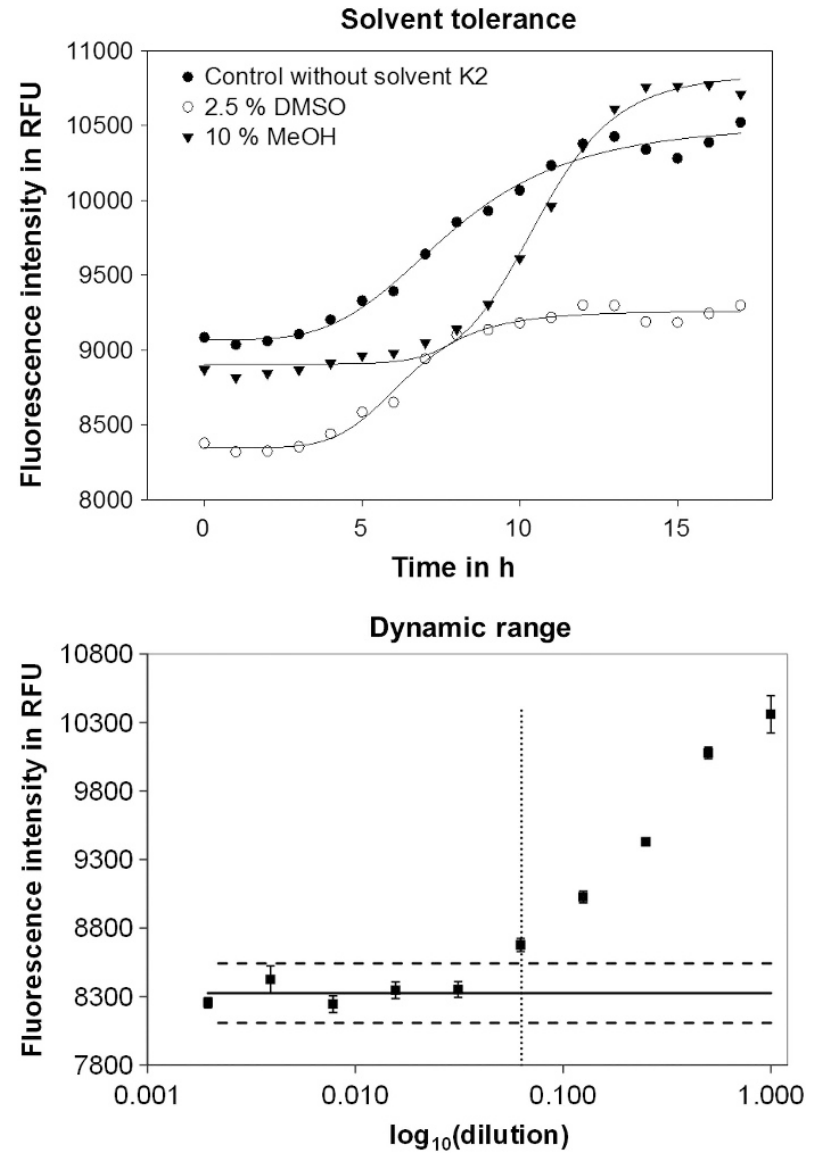

Figure 2 Influence of co-solvents methanol and dimethyl sulfoxide (DMSO) on the growth kinetics of $B$. subtilis (inoculation with $1.6 \times 10^{5}$ cells per $\mathrm{ml}$ ); relative standard errors of fluorescence measurements do not exceed $3 \%$ (for detailed growth curves, see Supplementary Figure S2 in Supplementary Information; up). Fluorescence measurements of bacterial cultures in several dilution steps with corresponding standard errors; horizontal solid line shows the fluorescence level of the mean of the five most diluted suspensions (1:512 to 1:32) with the dashed lines as the corresponding barrier of threefold the standard error of this mean; the vertical dotted line indicates the low end of the dynamic range at a dilution of ca 1:16 (down).

\section{Dynamic range}

To determine the low end of the dynamic range after an incubation time of $15 \mathrm{~h}$, the cultures were diluted with fresh medium containing $10 \%$ vol $\mathrm{MeOH}$ in 1:2 dilution steps until a dilution of 1:512 was reached. Subsequently, the fluorescence intensity of each dilution (six replicates for each) was measured (Figure 2, down). Although the upper limit is given by the end of the exponential growth phase, the lower end was defined at a value where fluorescence reaches below ca 3 -times of the standard error of autofluorescence (see dashed line in Figure 2, down) and above the autofluorescence base line of highly diluted bacterial suspension (see solid line in Figure 2, down). It was shown that the lower limit of the dynamic range is reached at a dilution of not much less than 1:16 (see dotted line in Figure 2, down), where the fluorescence signal is still distinguishable from the autofluorescence of media.

Comparison of the quantification methods based on fluorescence and OD

For the purpose of comparison between the growth inhibition assay based on fluorescence intensity with the one using OD measurements,
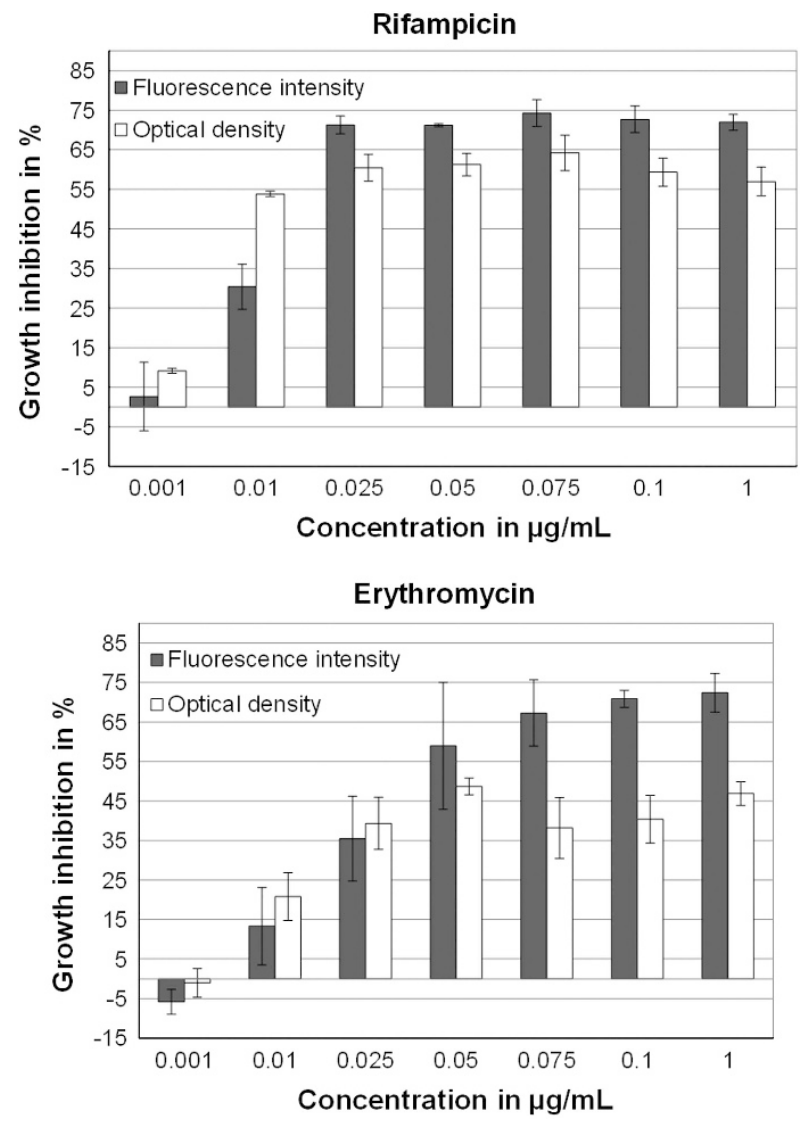

Figure 3 Comparison of fluorescence and OD detection methods to determine relative growth inhibition in \% of $B$. subtilis induced by different concentrations of rifampicin (up) and erythromycin (down) with s.e. of the mean.

the relative growth inhibition of the antibiotic compounds rifampicin and erythromycin were determined applying different concentrations (Figure 3). To quantify the bacterial growth after the incubation time, measurements of OD and fluorescence intensity were performed. A comparison of the growth inhibition induced by the two antibiotics indicates that both the fluorescence and the OD method give similar results (Figure 3 ).

Two conclusions can be drawn from the executed experiments. First, it is obvious that the growth inhibition values deduced from OD are lower than those determined by fluorescence detection in the range of higher concentrations of antibiotics $\left(0.025<\beta<1 \mu \mathrm{g} \mathrm{ml}^{-1}\right.$ for rifampicin and $0.05<\beta<1 \mu \mathrm{g} \mathrm{ml}^{-1}$ for erythromycin). In this special case, the inhibiting effect of a test compound may be underestimated in fluorescence assays compared with OD measurements. As the latter quantify all kinds of cells and cannot distinguish between living and dead cells or cell material, the fluorescence method, however, can be considered to provide a more realistic picture, as exclusively inhibition of growth is measured. Second, it is apparent from the comparison of both quantification methods that the standard deviation of the six replicates of the fluorescence measurement is approximately the same like the ones obtained by OD measurements. The fluorescence intensity measurements are based on the intrinsically produced YFP and therefore rely only on viable cells. Therefore, the findings demonstrate that the fluorescence quantification method to assess growth inhibition appears to be very precise, enabling an accurate determination of the bioactive effect with a similar overall sensitivity. 

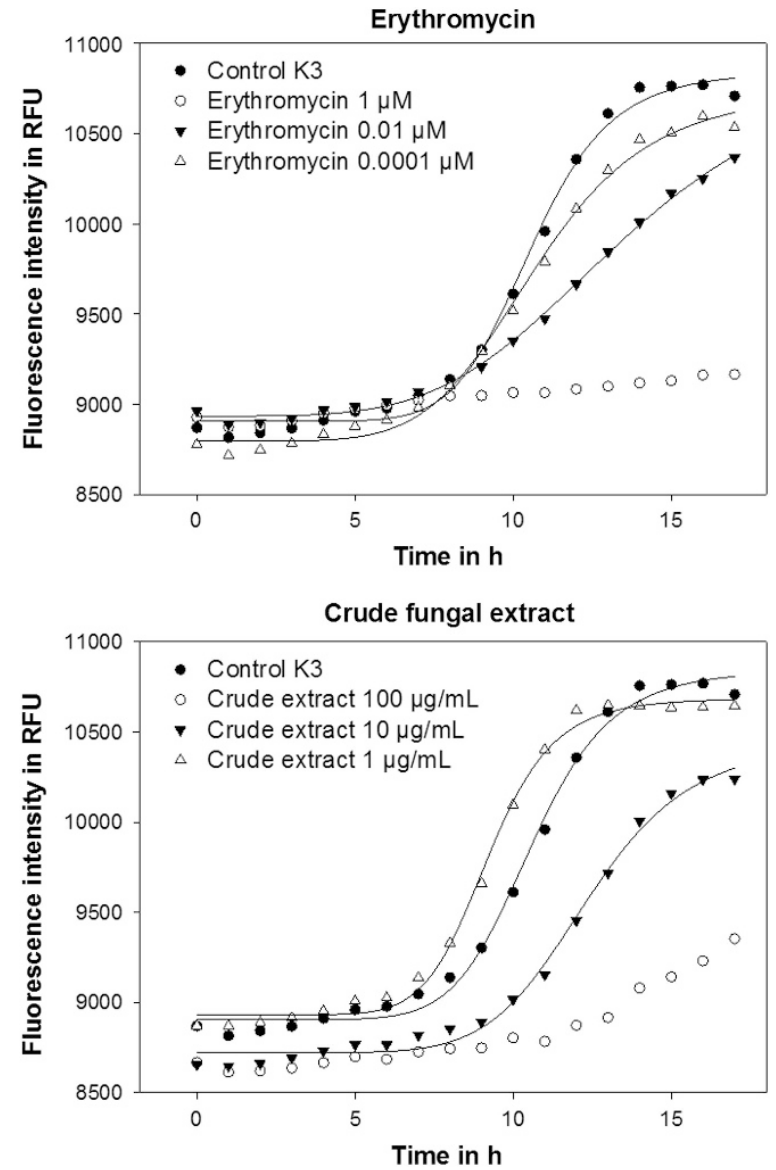

Figure 4 Growth kinetics of $B$. subtilis followed by fluorescence intensity measurements in the presence of different amounts of erythromycin (up) and a crude methanol extract from $H$. pustulatus (down) compared with control $\mathrm{K} 3$; relative standard errors of fluorescence measurements do not exceed $3 \%$ (detailed growth curves see Supplementary Figure 3 in Supplementary Information).

All in all, the concentration-dependent effect was induced in B. subtilis by both the bacteriostatic reagent erythromycin and the bactericidal compound rifampicin. This demonstrates that the assay is applicable for both types of antibacterial activity and does not discriminate between the two principle types of action. Since for a discovery screening of crude extracts and fractions, the identification of those extracts and fractions that contain bioactive compounds in general is required, this assay is suitable as a more general Grampositive antibacterial screening method. Later, distinct validation assays of eventually isolated hits should be made using established standard methods.

\section{Growth inhibition kinetics of crude extracts in comparison to erythromycin}

For validation, the growth kinetics of $B$. subtilis inhibited by different concentrations of a crude methanol extract of $H$. pustulatus, a fungus known to produce antibiotics, ${ }^{20}$ was compared with that of the standard antibiotic erythromycin (Figure 4).

First, it is clearly visible from Figure 4 that in both cases the bacterial growth is inhibited during the observed period of $17 \mathrm{~h}$ in comparison to control $\mathrm{K} 3$ (reference control with $\mathrm{MeOH}$ ). The inhibition by erythromycin is concentration-dependent because an increasing amount of the antibiotic results in a more reduced growth.

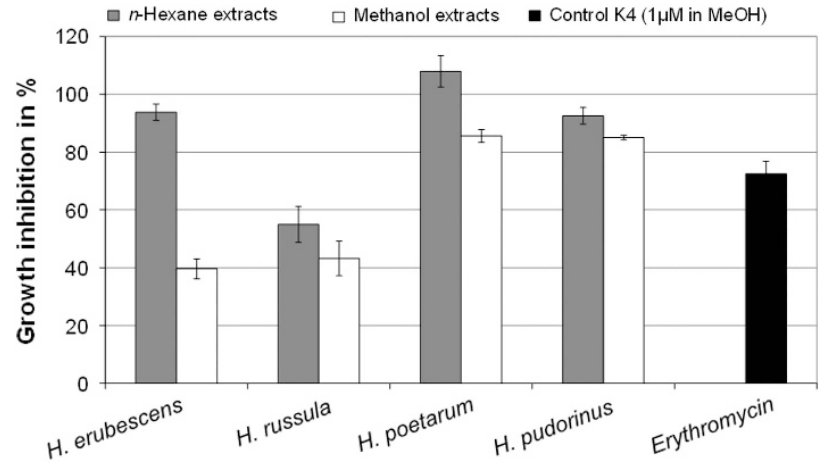

Figure 5 Bioactivities (relative growth inhibition of $B$. subtilis determined by fluorescence intensity measurements) of fungal extracts $\left(\beta=0.1 \mathrm{mg} \mathrm{ml}^{-1}\right.$ in $\mathrm{MeOH})$ after extraction with either pure methanol or $n$-hexane; erythromycin $(1 \mu \mathrm{m})$ as control $\mathrm{K} 4$.

The $\mathrm{Z}^{\prime}$-factor of ca 0.5 for the fluorescence data after the standard measuring time point of $15 \mathrm{~h}$ indicates a good quality of the assay. The same tendency of concentration-dependent inhibition was observed applying different concentrations of the crude methanol extract from $H$. pustulatus. Second, from the comparison of the growth curves it can be deduced that the starting point of the exponential growth phase is identical at all concentrations of erythromycin. The starting point of the exponential growth appears slightly postponed with increasing concentrations of the crude Hygrophorus extract. Most importantly, however, the assay can tolerate high extract concentrations. This is due to the fact that the indicator variable of fluorescence decreases with an increasing amount of bioactive compound and thus is not influenced by autofluorescence or errors in a false positive manner, that is, there is a counter-correlation of a potential artifact fluorescence and effectcoupled fluorescence. This is an advantage in comparison to other testing systems, where a high amount of extract and antibiotic correlates with higher fluorescence or OD (for example, tetrazolium salt assays). Increasing fluorescence or OD values may be also connected to more autofluorescent or colored products in highly concentrated extracts.

Application of the assay to screen crude extracts exemplified for the antibacterial activity of Hygrophorus extracts

Members of the fungal genus Hygrophorus produce a multitude of biologically active secondary metabolites. ${ }^{20-25}$ The determination of the bioactivity of crude extracts is a challenging task as the bioactive compound is embedded in a large excess of other components influencing both bacterial growth and the assessment of bioactivity. To evaluate the applicability of our assay, extracts of different Hygrophorus species were examined.

Effect of the extraction solvent on bioactivity. Different polarities of the extraction agent lead to compounds of the corresponding polarity in the extracted mixtures. Thus, it is reasonable to investigate if these differences are also reflected in bioactivity determined with the help of the growth inhibition assay. Therefore, the antibacterial activities of extracts with pure methanol were compared with the activities of extracts with non-polar $n$-hexane (Figure 5).

As a result, the non-polar $n$-hexane extracts exhibit higher antibiotic activities than the methanol extracts of the investigated species, but to a variable extent. Nevertheless, this indicates the increasing bioactive effect of these fungal extracts with decreasing polarity of the extraction solvent, which is not uncommon for compounds active, for example, in bacterial membranes. Furthermore, it demonstrates that the 


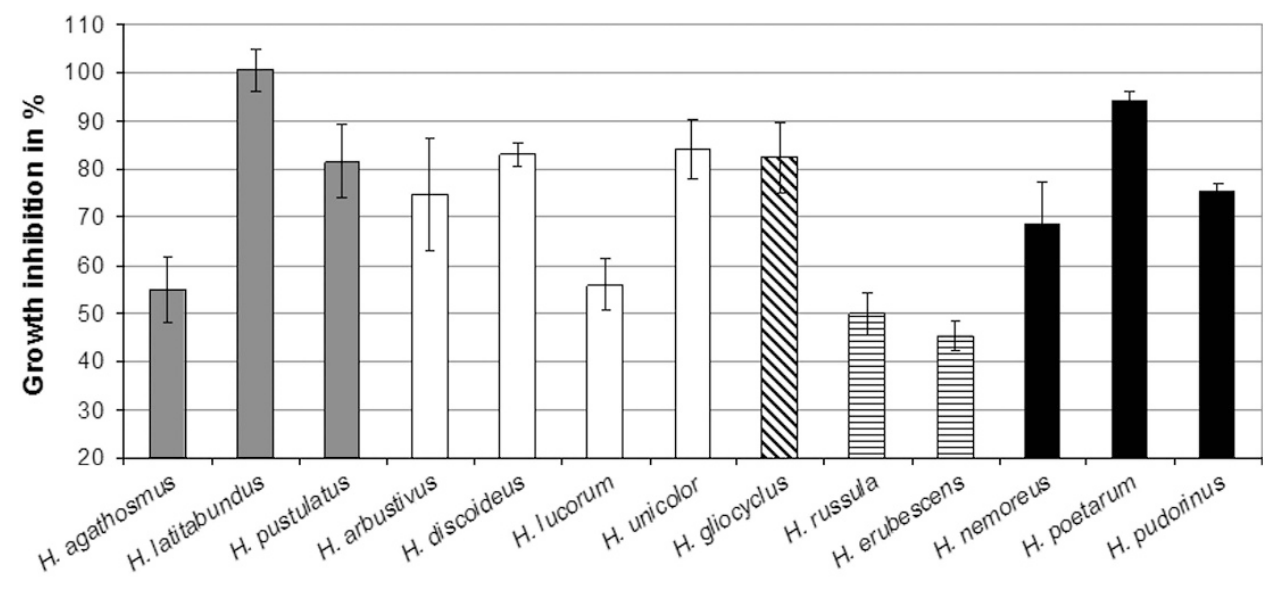

\section{$\square$ Section Olivaceoumbrini $\quad \square$ Section Discoidei $\mathbf{N}$ Section Hygrophorus \\ 目Section Erubescentes Section Pudorini}

Figure 6 Overview of the antibacterial properties (relative growth inhibition of $B$. subtilis determined by fluorescence intensity measurements) of different crude extracts (extraction agent: $100 \% \mathrm{MeOH} ; \beta=100 \mu \mathrm{g} \mathrm{ml}^{-1}$ ) of fruiting bodies from mushrooms of the genus Hygrophorus.
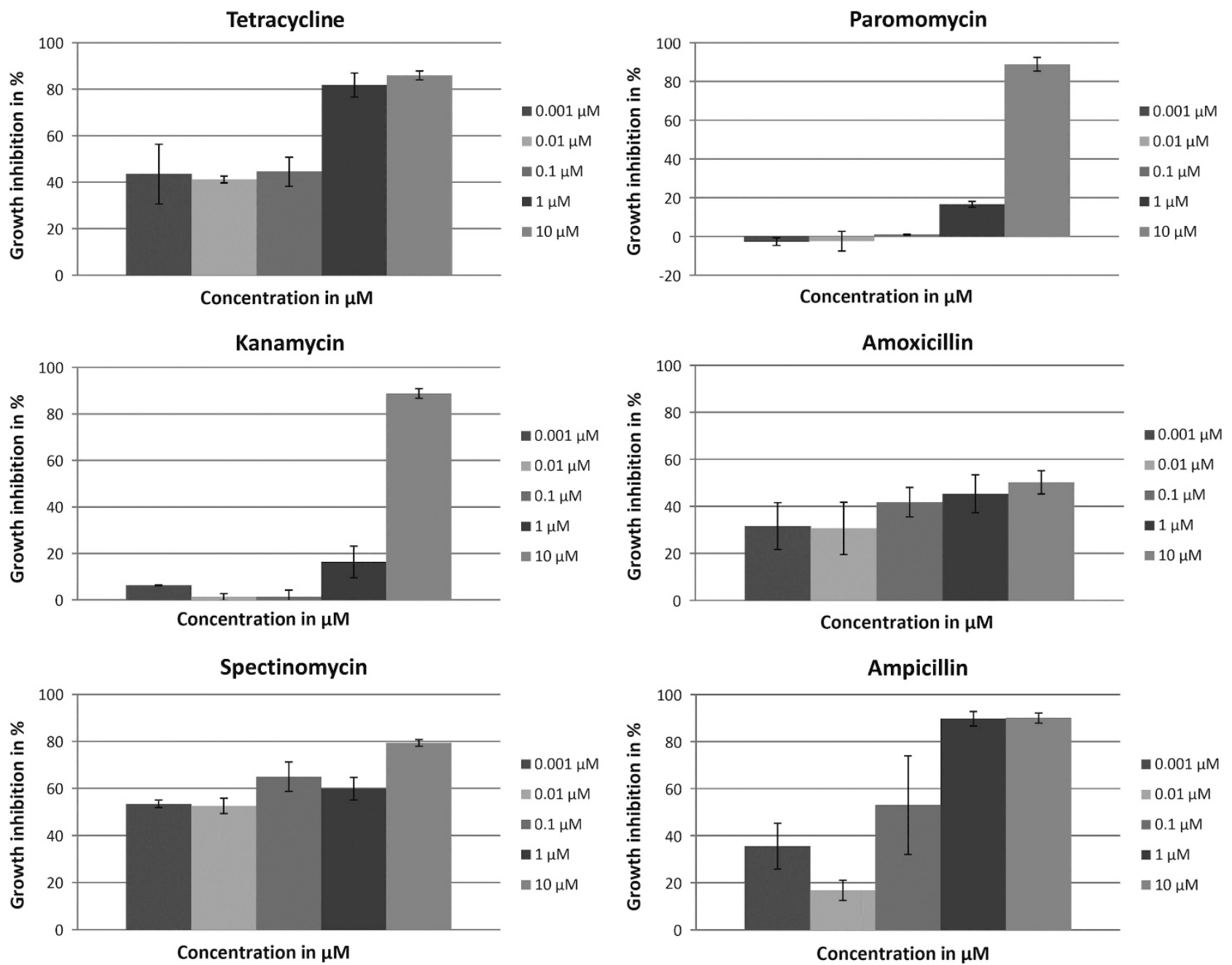

Figure 7 Relative growth inhibition of B. subtilis determined by fluorescence intensity measurements caused by tetracycline, paromomycin, kanamycin, amoxicillin, spectinomycin or ampicillin. 
fluorescence-based antibiotic assay developed herein is sensitive to differences in bioactivity caused by the nature of the extracted compound mixtures, which are accessible by variation of the extraction solvent.

Bioactivity screening of Hygrophorus species. Completing the investigation of the applicability of this assay, several crude methanol extracts of different Hygrophorus spp. from frozen fruiting bodies were screened for their antibacterial properties (Figure 6). The crude extracts of $H$. latitabundus and $H$. poetarum exhibit a remarkable bioactivity (ca 95-100\% inhibition at $\beta=100 \mu \mathrm{g} \mathrm{ml}^{-1}$ in $\mathrm{MeOH}$ ), whereas the extracts of $H$. russula and $H$. erubescens show a comparatively low activity with $43-50 \%$ relative inhibition (Figure 6).

\section{Assay response to different commercial antibacterial drugs}

Determination of $B$. subtilis growth inhibition by tetracycline, paromomycin, kanamycin, amoxicillin, spectinomycin and ampicillin was assessed using the newly developed fluorescence assay (Figure 7). Amoxicillin shows the lowest activity $(30-50 \%)$ in the investigated concentration range $(0.001-10 \mu \mathrm{M})$. The aminoglycoside antibiotics paromomycin and kanamycin strongly inhibited growth of B. subtilis in high concentration $(10 \mu \mathrm{M})$, whereas tetracycline and ampicillin (polyketide and $\beta$-lactam antibiotics, respectively) were also active at lower concentrations.

\section{CONCLUSION}

A rapid and robust bioassay for the quantitative determination of antibacterial activity is described. The assay is based on a derivative of Bacillus subtilis 168 (PabrB-iyfp), which produces an improved variant of the YFP during its exponential growth phase. This enables a very sensitive detection of bacterial growth undisturbed by dead cells or insoluble material. Interferences with colored or autofluorescent substances are reduced to a minimum, because extract amount and detection are inversely correlated and the fluorescence has specific excitation and emission wavelengths in a region usually unproblematic. It could be shown that the bioassay tolerates $\leqslant 2 \%$ vol DMSO, and $\mathrm{MeOH}$ even up to $10 \% \mathrm{vol}$, an important issue for the solubility of samples. To demonstrate its applicability in the field of natural product research and drug discovery, different types and amounts of crude extracts of Hygrophorus spp. were screened. Especially, the non-polar extracts employing $n$-hexane as eluent show a general and strong bioactive effect. Such lipophilic extracts are usually difficult to evaluate in standard assays ( $\leqslant 1 \%$ DMSO), but were handled well here in 10\% methanol.

The bioassay for antibacterials provides an elegant tool for the reliable, quantitative assessment of bioactive effects of both pure substances and complex mixtures such as crude extracts from natural sources. Thus, the presented fluorescence-based growth inhibition assay offers the opportunity to contribute to the search for natural product-based antibiotics since it can facilitate, for example, bioassayguided fractionation.

\section{CONFLICT OF INTEREST}

The authors declare no conflict of interest.

\section{ACKNOWLEDGEMENTS}

We thank Dr S Finsterbusch-Kettner, Dr GN Kaluđerović, C Würfel and $\mathrm{K}$ Mißbach for assistance. Furthermore, we gratefully acknowledge the Leibniz Association for financial support as part of the SAW-Project 'Identifizierung eigenschaftsrelevanter Metabolitencluster'.

1 Abraham, E. P. et al. Further observations on penicillin. Lancet 238, 177-189 (1941)

2 Bauer, A. W., Kirby, W. M. M., Sherris, J. C. \& Turck, M. Antibiotic susceptibility testing by a standardized single disk method. Am. J. Clin. Pathol. 45, 493-496 (1966).

3 Vincent, J. G. \& Vincent, H. W. Filter paper disc modification of the oxford cup penicillin determination. Proc. Soc. Exp. Biol. Med. 55, 162-164 (1944).

4 Finn, R. K. Theory of agar diffusion methods for bioassay. Anal. Chem. 31, 975-977 (1959).

5 Casey, J. T., O'Cleirigh, C., Walsh, P. K. \& O'Shea, D. G. Development of a robust microtiter plate-based assay method for assessment of bioactivity. J. Microbiol. Meth 58, 327-334 (2004).

6 Rufián-Henares, J. A. \& Morales, F. J. A new application of a commercial microtiter plate-based assay for assessing the antimicrobial activity of Maillard reaction products. Food Res. Int. 39, 33-39 (2006).

7 Singh, U., Akhtar, S., Mishra, A. \& Sarkar, D. A. novel screening method based on menadione mediated reduction of tetrazolium salt for testing of antimycobacterial agents. J. Microbiol. Meth. 84, 202-207 (2011).

8 Tengerdy, R. P., Nagy, J. G. \& Martin, B. Quantitative measurement of bacterial growth by reduction of tetrazolium salts. Appl. Microbiol. 15, 954-955 (1967).

9 Wanandy, S. et al. Optimisation of the fluorescein diacetate antibacterial assay. J. Microbiol. Meth. 60, 21-30 (2005).

10 Casey, W. M. \& Nguyen, N. T. Use of the green fluorescent protein to rapidly assess viability of Escherichia coli in preserved solutions. J. Pharm. Sci. Technol. 50, 352-355 (1995).

11 Webb, J. S. et al. Green fluorescent protein as a novel indicator of antimicrobial susceptibility in Aureobasidium pullulans. Appl. Environ. Microbiol. 67, 5614-5620 (2001).

12 Veening, J. W., Smits, W. K., Hamoen, L. W., Jongbloed, J. D. H. \& Kuipers, O. P. Visualization of differential gene expression by improved cyan fluorescent protein and yellow fluorescent protein production in Bacillus subtilis. Appl. Environ. Microbiol. 70, 6809-6815 (2004).

13 Harwood, C. R. \& Wipat, A. Sequencing and functional analysis of the genome of Bacillus subtilis strain 168. FEBS Lett. 389, 84-87 (1996).

14 Strauch, M. A. et al. Abh and AbrB control of Bacillus subtilis antimicrobial gene expression. J. Bacteriol. 189, 7720-7732 (2007).

15 Veening, J. W., Kuipers, O. P., Brul, S., Hellingwerf, K. J. \& Kort, R. Effects of phosphorelay perturbations on architecture, sporulation, and spore resistance in biofilms of Bacillus subtilis. J. Bacteriol. 188, 3099-3109 (2006).

16 Zhang, J.-H., Chung, T. D. Y. \& Oldenburg, K. R. A. Simple statistical parameter for use in evaluation and validation of high throughput screening assays. J. Biomol. Screen. 4, 67-73 (1999).

17 Blom, E. J., Ridder, A. N., Lulko, A. T., Roerdink, J. B. \& Kuipers, O. P. Time-resolved transcriptomics and bioinformatic analyses reveal intrinsic stress responses during batch culture of Bacillus subtilis. PLoS One 6, e27160 (2011).

18 Thomsen, $\mathrm{H}$. et al. Characterization of constituents and anthelmintic properties of Hagenia abyssinica. Sci. Pharm. 80, 433-446 (2012).

19 Kataoka, N. et al. Development of butanol-tolerant Bacillus subtilis strain GRSW2-B1 as a potential bioproduction host. AMB Express 1, 10(1-11) (2011).

20 Lübken, T., Schmidt, J., Porzel, A., Arnold, N. \& Wessjohann, L. Hygrophorones A-G fungicidal cyclopentenones from Hygrophorus species (Basidiomycetes). Phytochemistry 65, 1061-1071 (2004).

21 Eschen-Lippold, L. et al. Antioomycete activity of $\gamma$-oxocrotonate fatty acids against P. infestans. J. Agric. Food Chem. 57, 9607-9612 (2009).

22 Gilardoni, G., Clericuzio, M., Tosi, S., Zanoni, G. \& Vidari, G. Antifungal acylcyclopentenediones from fruiting bodies of Hygrophorus chrysodon. J. Nat. Prod. 70, 137-139 (2006).

23 Gilardoni, G. et al. New oxidized 4-oxo fatty acids from Hygrophorus discoxanthus. Nat. Prod. Commun. 1, 1079-1084 (2006).

24 Lübken, T., Arnold, N., Wessjohann, L., Böttcher, C. \& Schmidt, J. Analysis of fungal cyclopentenone derivatives from Hygrophorus spp. by liquid chromatography/ electrospray-tandem mass spectrometry. J. Mass Spectrom. 41, 361-371 (2006).

25 Teichert, A. et al. Unusual bioactive 4-oxo-2-alkenoic fatty acids from Hygrophorus eburneus. Z. Naturforschung 60b, 25-32 (2005).

Supplementary Information accompanies the paper on The Journal of Antibiotics website (http://www.nature.com/ja) 